\title{
Innover dans le secteur de la transformation agroalimentaire en Afrique de l'Ouest
}

\author{
Thierry Ferré ${ }^{1,5, *}$, Ignace Medah ${ }^{2}$, Jean-François Cruz ${ }^{3,5}$, Marie-Hélène Dabat ${ }^{4,5}$, Pierre-Yves Le Gal ${ }^{1,5}$, \\ Matthieu Chtioui ${ }^{6}$ et Agathe Devaux-Spatarakis ${ }^{7}$ \\ ${ }^{1}$ CIRAD, UMR Innovation, 34398 Montpellier, France \\ 2 IRSAT/CNRST, Ouagadougou, Burkina Faso \\ ${ }^{3}$ CIRAD, UMR Qualisud, 34398 Montpellier, France \\ ${ }^{4}$ CIRAD, UMR ART-DEV, 34398 Montpellier, France \\ ${ }^{5}$ Univ Montpellier, Montpellier, France \\ ${ }^{6}$ ISTOM, Cergy, France \\ ${ }^{7}$ QUADRANT CONSEIL, Paris, France
}

\begin{abstract}
Résumé - L'article analyse, à l'aide de la méthode ImpresS conçue par le Cirad, un processus d'innovation impulsé par les utilisatrices d'un équipement de transformation: le décortiqueur et blanchisseur de fonio (Digitaria exilis Stapf) en Afrique de l'Ouest. L'identification du système d'acteurs et le récit chronologique du processus d'innovation ont été mobilisés pour caractériser les mécanismes et les conditions qui expliquent la réussite de cette innovation. Ils mettent en évidence le rôle déterminant d'un partenariat autour d'objectifs partagés entre plusieurs acteurs (chercheurs, transformatrices et équipementiers) et l'importance de la mobilisation d'un capital de connaissances chez ces acteurs pour résoudre un problème sociétal: la pénibilité du décorticage du fonio. Les apprentissages croisés, formels ou informels, entre les parties prenantes, ont été essentiels à l'émergence de solutions aux problèmes rencontrés en cours de processus.
\end{abstract}

Mots clés : processus d'innovation / innovation / apprentissage / fonio / Burkina Faso / Mali

Abstract - Innovating in the agri-food processing sector in West Africa. Based on the ImpresS method developed by CIRAD, the article analyzes the innovation processes driven by the users of the fonio (Digitaria exilis Stapf) dehuller in West Africa. The characterization of the actors system and the chronological narrative of the innovation process were mobilized to characterize the mechanisms and conditions explaining the success of this innovation. They highlight the decisive role of building a partnership around shared objectives between several stakeholders (researchers, processors and equipment manufacturers) and the importance of mobilizing a capital of knowledge among these stakeholders to solve a societal problem: the drudgery of hulling fonio. Formal and informal cross learning between stakeholders were essential in solving problems encountered during the process.

Keywords: innovation process / learning / fonio / Burkina Faso / Mali

\section{Introduction}

De nombreuses tentatives d'innovations techniques dans la transformation des produits agricoles en Afrique de l'Ouest se sont révélées infructueuses, mais ces échecs ont toutefois contribué à faire évoluer les démarches et les méthodes de conception d'équipements (Bricas et Raoult-Wack, 1997;

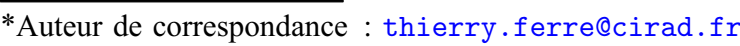

Marouzé et al., 2004 ; Fofiri et al., 2015). Force est de constater que ces avancées méthodologiques ont cependant eu peu d'impact sur l'offre des équipementiers de cette région (Giroux et Marouzé, 2006). La production locale d'équipements ne satisfait que très partiellement aux demandes des petites et moyennes entreprises (PME) qui veulent mécaniser certaines tâches manuelles, véritables goulets d'étranglement dans le processus de transformation des produits (Bricas, 2012). Les faibles capacités financières de ces PME comme les difficultés d'accès aux financements (Nguena, 2013) limitent aussi le 
recours aux importations d'équipements. Il s'avère donc stratégique d'agir sur le renforcement des capacités locales de conception et de production d'équipements afin de pourvoir aux besoins de ces PME agroalimentaires.

Le décorticage et le blanchiment du fonio (Digitaria exilis Stapf) représentent un cas emblématique de cette situation. Comme le riz, le fonio est une céréale vêtue appelée «fonio paddy », dont les grains sont entourés de balles (les glumes et glumelles). Le décorticage permet, par élimination des balles, d'obtenir le fonio décortiqué ou fonio complet. Puis le blanchiment, par élimination du péricarpe et du germe (son) des grains, donne le fonio blanchi qui est la principale forme de consommation (Cruz, 2004). Ces opérations sont traditionnellement réalisées par les femmes au pilon et au mortier. Elles constituent des tâches manuelles pénibles qui nécessitent près d'une heure pour transformer de 1 à $3 \mathrm{~kg}$ de grains. Jusqu'à l'arrivée d'équipements mécanisés, ces opérations constituaient de puissants freins au développement du fonio et entraînaient son déclin dans de nombreuses zones de production. Un premier équipement, le décortiqueur « Sanoussi », a été conçu et fabriqué au Sénégal au début des années 1990, mais la plupart des utilisatrices l'ont abandonné du fait de performances techniques jugées insuffisantes. À partir de 2002, il a été remplacé par un équipement appelé décortiqueur « GMBF » pour « Guinée, Mali, Burkina Faso, France», conçu par le Cirad et ses partenaires africains. Cet équipement, qui fonctionne selon le principe des décortiqueurs à riz de type «Engelberg », a été adapté à la très petite taille des grains de fonio $(1,8 \mathrm{~mm}$ de long et $0,9 \mathrm{~mm}$ de large). Le décortiqueur GMBF a une capacité de 50 à $100 \mathrm{~kg} / \mathrm{h}$ et un rendement global de transformation de l'ordre de $65 \%$ (Cruz et al., 2011).

Le décortiqueur GMBF a connu une large diffusion au Mali, avec plus de 150 unités produites principalement par deux fabricants, IMAF (Industries Mali flexibles) et SIPS (Société industrielle des prestations de service), contre une quinzaine au Burkina Faso. Mais le nombre total de machines produites au Mali est sans doute bien plus important avec l'existence de nombreuses machines calquées sur le modèle GMBF. En 2014, lors de nos enquêtes, plus de 70 entreprises de transformation de fonio ont été recensées dans la seule ville de Bamako, dont les trois quarts ont été créées dans les années 2000. Le décortiqueur GMBF a également permis d'accroître largement la production des quatre entreprises de transformation qui ont participé à sa mise au point au Mali et au Burkina Faso. En moins de quinze ans, elles sont passées d'une petite production annuelle de quelques centaines de kilogrammes de fonio transformé à des volumes annuels variant de 50 à $200 \mathrm{t}$ (Ferré et al., 2016).

La diffusion du décortiqueur GMBF dans les villages maliens est à l'origine d'un accroissement des surfaces cultivées en fonio et du nombre d'exploitations pratiquant cette culture. Cette dynamique est confirmée par les statistiques de la FAO, qui donnent pour le Mali une hausse de la production annuelle de fonio de 16000 à plus de $50000 \mathrm{t}$ sur la période 2002 à 2011 et une production moyenne actuelle supérieure à $30000 \mathrm{t}$ alors que la production était auparavant en déclin (http://www. fao.org/faostat/fr/\#data/QC). Les $\mathrm{ONG}$ et les projets de développement sont intervenus au cours de la phase de diffusion de l'équipement comme facilitateurs (subventions) et prescripteurs, alors que l'équipement était déjà solidement confirmé par les opérateurs économiques. Cette implication des ONG et des
États a eu lieu relativement tard, lorsque la diffusion de l'équipement était déjà bien engagée.

Cet article vise à comprendre les processus qui ont abouti à la large diffusion de cet équipement, en se focalisant sur les mécanismes ayant conduit aux impacts observés et aux chaînes de traduction des connaissances à l'œuvre à travers les interactions entre acteurs. La méthode ImpresS, pour « IMPact des REcherches au Sud» (Temple et al., 2016; Barret et al., 2017), est utilisée à cet effet. Elle permet à la fois de comprendre le processus d'innovation, de rendre compte des liens de causalité qui conduisent aux impacts et d'évaluer les contributions des différentes parties prenantes, dont la recherche. Nous présentons en premier lieu les outils utilisés, puis les étapes structurantes du processus d'innovation. Nous discutons ensuite des contributions de la recherche et des autres parties prenantes à l'innovation dans le cas du décortiqueur de fonio, et plus globalement de la conception d'innovation en partenariat dans les pays en développement d'Afrique de l'Ouest.

\section{Cadre méthodologique}

Cette recherche fait le récit de la conception en partenariat du décortiqueur de fonio et analyse les interactions entre les principales parties prenantes de trois pays: la France, le Burkina Faso et le Mali. Les données et informations de base ont été collectées à partir d'observations directes, d'entretiens semi-directifs et d'analyses documentaires. L'objectif était d'obtenir une meilleure connaissance des interactions sociales et de leurs enjeux, des représentations et des pratiques des acteurs.

Les enquêtes se sont déroulées dans les villes de Bamako, Ouagadougou et Bobo-Dioulasso où existe une forte concentration d'entreprises de transformation du fonio, ainsi que dans ses principales zones de production. L'élaboration du réseau d'acteurs rencontrés a suivi une approche constructiviste fondée sur les associations et les dissociations révélées par les acteurs au fil de nos rencontres. Cette méthode dépourvue d'échantillonnage préalable n'a donc aucune ambition de représentativité statistique. Pour autant, une grande diversité d'acteurs a été mobilisée, touchant directement ou indirectement à l'innovation et à la filière fonio : chercheurs, ONG, producteurs, transformatrices, équipementiers, commerçants, prestataires de services (Tab. 1). Au final, 186 individus ont été interrogés lors d'entretiens individuels et de focus groupes et 84 au cours d'ateliers réunissant des panels d'acteurs.

L'étude s'appuie également sur de nombreux documents écrits: rapports scientifiques et techniques, documents de formation, fiches techniques, dossiers de fabrication. Ces documents sont principalement issus du projet de recherche «Amélioration des technologies post-récolte du fonio» financé par le CFC (Common Fund for Commodities), coordonné par le Cirad de 1999 à 2004, supervisé par la FAO et réalisé en collaboration avec l'Institut de recherche agronomique de Guinée (IRAG), l'Institut d'économie rurale (IER) du Mali et l'Institut de recherche en sciences appliquées et technologies (IRSAT) du Burkina Faso.

L'ensemble des éléments recueillis a été analysé pour mettre en évidence les relations et les modes de collaboration 
Tableau 1. Les outils de collecte des données.

Table 1. Data collection tools.

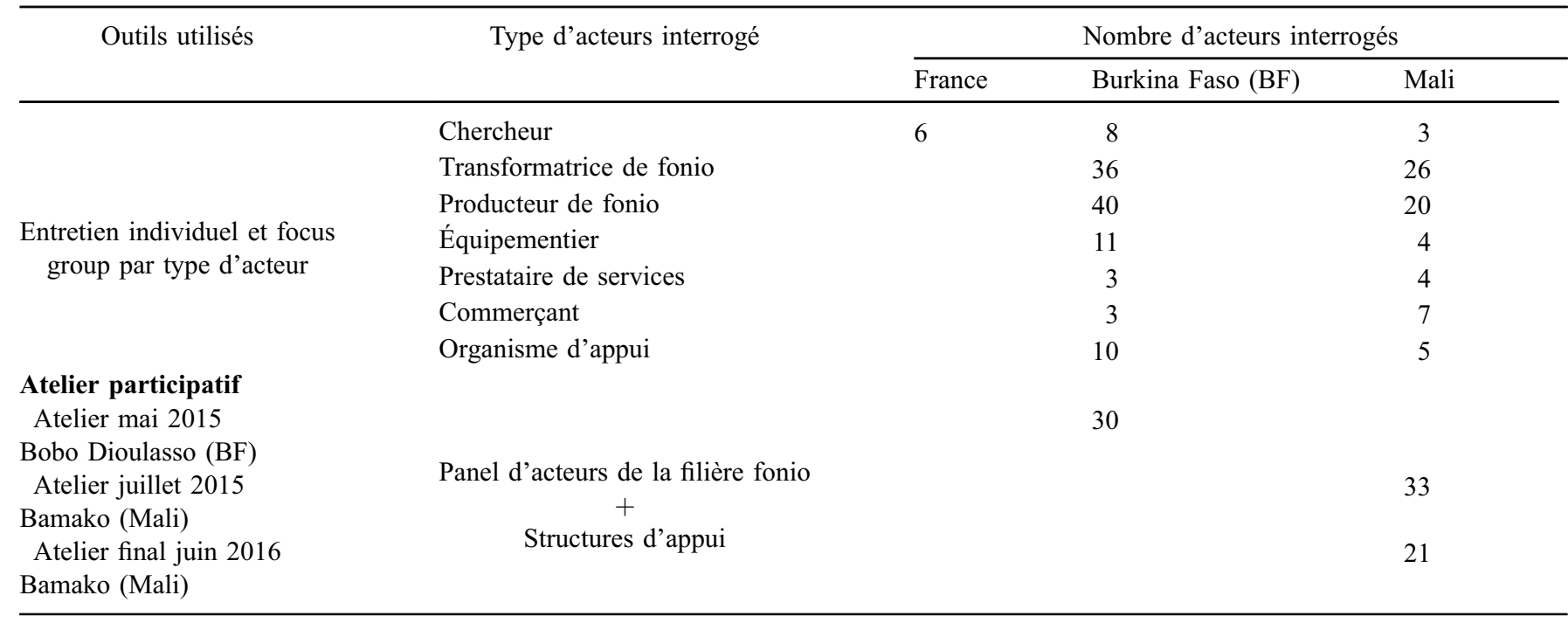

qui associent la recherche technologique, les acteurs du secteur agroalimentaire et les autres parties prenantes du processus.

\section{Les spécificités du processus d'innovation}

\subsection{Politiques incitatives et construction d'un réseau d'acteurs comme préalables au processus d'innovation technique}

La fin des années 1980 et le début des années 1990 sont marqués par des initiatives qui visent à promouvoir les céréales locales au Sahel et à renforcer les initiatives des acteurs de la transformation agroalimentaire. Suite aux recommandations du colloque de Mindelo de 1986 portant sur les politiques céréalières dans les pays du Sahel et prônant de «Transformer sahélien pour consommer sahélien», le Comité permanent inter-États de lutte contre la sécheresse dans le Sahel (Cilss) et le Club du Sahel décident de mettre en place le Programme régional de promotion des céréales locales au Sahel (Procelos) qui se déroule de 1994 à 2005 et dont Mme M. devient animatrice pour le Mali. Des chercheurs sont présents dans différentes instances de concertation où se discutent les problématiques de développement du secteur agroalimentaire et de la valorisation des céréales locales. Ils y côtoient les acteurs publics comme privés et deviennent partie prenante de l'élaboration du programme Procelos qui rassemble Enda (Environnement et développement du tiers monde) et le groupe Altersial (Alternatives techniques et systèmes alimentaires), constitué de l'Ensia (École nationale supérieure des industries agricoles et alimentaires), du Cirad et du Gret.

L'impulsion du processus d'innovation dans le cas du fonio est venue de transformatrices de céréales qui, telle Mme M., sont considérées par leurs pairs et par les autorités comme des leaders de 1'entrepreneuriat agroalimentaire. En 1993, deux transformatrices du Mali et du Burkina Faso convainquent les chercheurs du Cirad de s'intéresser à la mécanisation du décorticage du fonio. L'une d'elles, la même Mme M. du Mali, occupe alors des fonctions de représentation des professionnels du secteur de la transformation des céréales. En 1994, lors de colloques régionaux à Dakar puis à Bamako, elle fait un état des lieux critique de la situation des PME agroalimentaires du Mali et réclame différentes mesures en matière d'accompagnement et de soutien au développement du secteur de la transformation des céréales. Elle revendique que les problèmes relatifs à la transformation du fonio, notamment le décorticage, soient urgemment pris en compte par les institutions régionales et la recherche.

Lors du colloque régional de Bamako, sont présentés les résultats des premiers essais de décorticage mécanique du fonio réalisés au Cirad sur un prototype de décortiqueur à mil et sorgho, le DMS 500. Les échanges entre les transformatrices et les chercheurs sur le fonio aboutissent à la modification du principe de décorticage et à l'élaboration d'un nouveau cahier des charges fonctionnel. Au-delà des discussions techniques, ces rencontres entre transformatrices et chercheurs marquent un tournant dans l'histoire de la mécanisation des opérations de transformation du fonio. Les chercheurs du Cirad inscrivent cette question à leur agenda et deviennent désormais des porteparoles auprès de leurs pairs d'Afrique de l'Ouest, des décideurs politiques et des bailleurs de fonds. L'enrôlement des chercheurs constitue une importante avancée stratégique pour les transformatrices de fonio.

Ce dialogue entre transformatrices et chercheurs préfigure la démarche de co-conception portée par le projet qui s'inspire avant tout de la méthode CESAM (Conception d'équipements dans les pays du Sud pour l'agriculture et l'agroalimentaire, méthode) développée par le Cirad (Marouzé, 1999) en réponse aux nombreux cas d'échecs de transfert de technologies observés par les chercheurs. Ceux-ci perçoivent les limites des approches diffusionnistes reposant sur la mise au point d'équipements ou de procédés nouveaux en laboratoire puis leur transfert aux utilisateurs. La nécessité de formaliser une démarche spécifique de conception qui tienne compte du contexte des pays du Sud apparaît indispensable. Il s'agit surtout d'intégrer plus précocement et plus précisément à la 
fois les conditions locales de fabrication (fabricabilité) et les besoins des futurs utilisateurs. Cette méthode de conception applicable au domaine des équipements à petite échelle dans les secteurs agricole, agroalimentaire et énergétique, est initiée à l'occasion d'un séminaire international organisé en 1996 à Montpellier, qui rassemble une douzaine de concepteurs originaires de sept pays du Sud. La démarche consigne un certain nombre de passages obligés, dont la validation conditionne le processus de conception.

L'entreprise IMAF, fabricant d'équipements installé à Bamako, est le dernier acteur clé à rejoindre le collectif de recherche en 2001. Son ralliement au dispositif est formalisé par une «convention associée» avec le Cirad et l'IER. Cette convention fixe les modalités de collaboration et de mise en œuvre de la fabrication par IMAF du décortiqueur de fonio. L'enrôlement dans le dispositif du responsable de cette entreprise est pour partie lié aux relations interpersonnelles qu'il avait nouées avec les chercheurs de l'IER et du Cirad basés à Bamako. Après évaluation de ses capacités techniques, l'entreprise est sélectionnée pour être le principal équipementier partenaire du projet au Mali. Le premier prototype de décortiqueur à fonio est fabriqué en 2001 par IMAF sous la supervision d'un chercheur du Cirad. Avec l'arrivée de cet acteur se trouve ainsi constitué le collectif au cœur du processus de conception, composé principalement des chercheurs, des transformatrices et de l'équipementier. Un deuxième équipementier, l'entreprise SIPS, a rejoint l'équipe du projet en 2003 .

\subsection{La production et la diffusion des connaissances dans le processus d'innovation}

L'intérêt des chercheurs du Cirad pour le décorticage du fonio rejoint leurs préoccupations plus anciennes et plus générales concernant la mécanisation des opérations postrécolte et de première transformation des céréales. Dès les années 1980, le département de machinisme agricole du Cirad collabore sur ce thème avec de nombreux centres de recherche d'Afrique de l'Ouest. Une communauté de chercheurs se constitue, partageant une problématique commune liée aux opérations de première transformation des céréales et notamment au décorticage (Abécassis et al., 1980). À la fin des années 1980 et au début des années 1990, les chercheurs d'Afrique de l'Ouest et du Cirad mettent au point différents équipements de transformation des céréales, notamment pour le riz (Bergeret et al., 1989), le mil et le sorgho. Grâce à ces différents travaux, les chercheurs produisent des connaissances spécifiques sur le décorticage et des connaissances contextualisées sur la conception des équipements, leur fabrication, leur utilisation et leur maintenance (Cruz et Havard, 1994). Elles constituent des ressources mobilisables dans d'autres situations pour répondre à de nouvelles questions de recherche.

Ces connaissances ont été mobilisées dans le cas du fonio avec les principales parties prenantes du processus d'innovation (transformatrices, équipementiers) concernées par le renforcement des capacités. Cette dimension s'est notamment avérée essentielle chez l'équipementier IMAF de Bamako. Dans cette entreprise, la transmission des compétences s'est faite principalement via le chef d'atelier. L'atelier y est le principal lieu d'apprentissage, même si aucune formation formelle n'y a été dispensée. Le mode de transmission des savoirs, des savoir-faire et des compétences s'apparente à de la formation «sur le tas » ou à de l'apprentissage non formel. Le renforcement de capacités s'est accompli chemin faisant, dans un processus d'échanges et d'interactions relativement long (trois ans) entre les chercheurs et le personnel de l'atelier d'IMAF (Fig. 1). Les situations d'apprentissage sont survenues tout au long du processus, essentiellement lors de la résolution de problèmes concrets : lecture de plans, traçage de pièces, choix de matériaux, usinage de pièces, assemblage, etc. Cet apprentissage non formel est également intervenu lors des essais de machines en milieu réel, dans les entreprises ou dans les villages. Là encore, les mécaniciens d'IMAF interagissaient avec les chercheurs mais également avec les transformatrices, apprenaient à résoudre des problèmes en équipe et dans des situations variées.

L'intervention de la recherche s'est poursuivie après la fin du projet, principalement à travers des actions de formation destinées aux transformatrices, mais également par la mise à disposition du décortiqueur (Fig. 1). Ainsi, à partir de 2008, un exemplaire du décortiqueur GMBF est accessible en prestation de services au sein même de la station de recherche de l'IER à Bamako. Cette phase d'utilisation en routine a permis aux techniciens et chercheurs de l'IER de mieux maîtriser l'utilisation de l'équipement et sa maintenance. Ce fut également l'opportunité, pour les entreprises de Bamako non équipées, d'avoir accès au décortiqueur et ainsi de s'assurer des performances effectives de l'équipement. Cette étape correspond à un moment clé du processus d'innovation, au cours duquel la fiabilité de l'équipement a été validée auprès de nombreuses utilisatrices futures. Cette séquence a contribué à asseoir la crédibilité de l'équipementier et des chercheurs auprès des transformatrices, en faisant connaître le décortiqueur et en facilitant son accès à un plus grand nombre d'entre elles.

Le changement d'échelle observé lors du déploiement de l'innovation s'est traduit, tant au Mali qu'au Burkina Faso, par l'émergence de prestataires privés dans les villes, les petits centres urbains et les zones rurales. Ce nouveau service a libéré les femmes d'une tâche très pénible et a contribué à la relance de la production de fonio (Ferré et al., 2016). La diffusion de ce décortiqueur est cependant bien moins importante au Burkina Faso, où les décortiqueurs proviennent du Mali, sans doute parce que l'institution de recherche burkinabé n'avait pas jugé utile d'impliquer les artisans locaux et les transformatrices au processus.

\section{Discussion}

La mobilisation de la méthode ImpresS pour l'étude de la conception et de la diffusion d'un décortiqueur de fonio en Afrique de l'Ouest a permis de repérer plusieurs facteurs explicatifs de la réussite d'un processus d'innovation dans le secteur de l'équipement agroalimentaire dans les pays du Sud. L'approche collaborative en partenariat, impliquant des institutions de recherche du Nord et du Sud, des transformatrices de céréales utilisatrices des équipements et des artisans/ équipementiers locaux, a fourni le cadre organisationnel permettant de faciliter les apprentissages croisés. Ce cadre a 


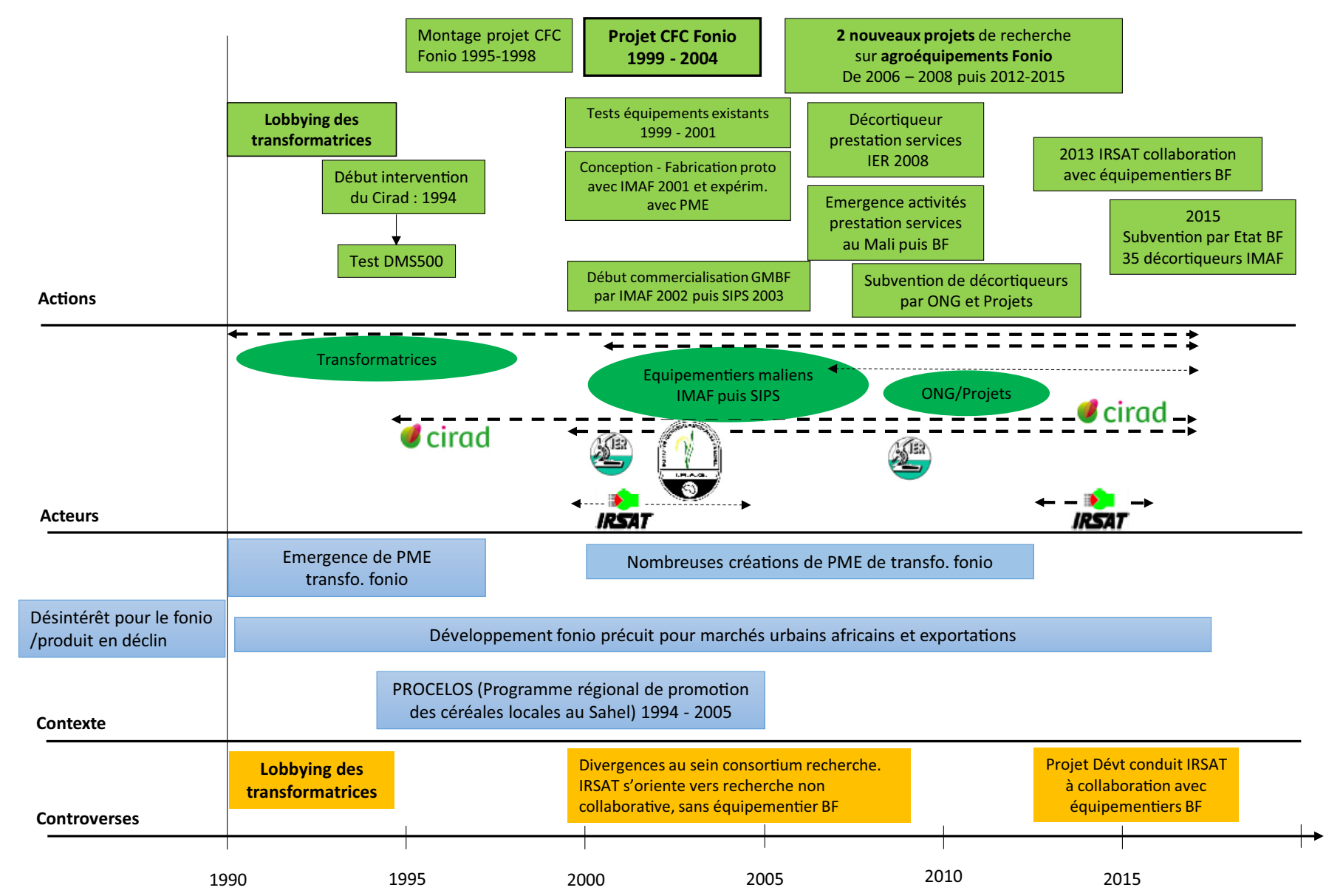

Fig. 1. Chronogramme historique de l'innovation «décortiqueur fonio».

Fig. 1. Historical chronogram of the "fonio dehuller" innovation.

bénéficié pour un temps de l'existence d'un projet formel, mais a également fonctionné sous un mode informel. Il se rattache donc pour une part aux plateformes d'innovation, considérées aujourd'hui comme un lieu, physique ou social, d'interactions entre les différents acteurs impliqués dans un processus d'innovation (Adjei-Nsiah et Klerkx, 2016).

Les acteurs insérés dans ce cadre ont produit et échangé un ensemble de connaissances dans un processus d'exploration et d'innovation autour de la transformation du fonio et plus largement des céréales locales. Ces connaissances concernent tant les savoirs scientifiques construits par les chercheurs que les savoirs empiriques accumulés par les transformatrices et les équipementiers. Cette hybridation des connaissances (MoityMaïzi, 2011) a été centrale pour trouver des solutions aux problèmes rencontrés en cours de processus.

Ce dispositif a également profité de la démarche de conception mobilisée dès le démarrage du projet. Inspirée de démarches utilisées dans le secteur industriel (Blanco, 1998), la méthode CESAM a permis la prise en compte des contraintes de la conception et de la vie du produit depuis la mobilisation des compétences nécessaires à la mise au point de l'innovation, l'enrôlement réussi des acteurs et le maintien en veille de la dynamique interne des interactions des différentes parties prenantes.

In fine, l'ensemble du processus a favorisé le renforcement des capacités et la construction d'un capital humain et social au sein du réseau d'acteurs impliqués. Ce constat corrobore les principes de la méthode ImpresS qui fait du renforcement des capacités un des piliers de la démarche dans sa contribution au chemin de l'impact (Temple et al., 2016). Ainsi, le partage entre pairs d'une méthodologie commune en matière de conception d'équipements a permis aux chercheurs d'enrichir mutuellement leurs connaissances et la mise en œuvre d'une certaine transdisciplinarité entre génie des procédés, génie industriel et sciences sociales (Sardas, 1997). Les interactions entre les parties prenantes clés du dispositif innovant, les transformatrices, les équipementiers et les chercheurs, tout au long du processus, ont contribué au renforcement des capacités à innover. Par la suite, elles ont aussi facilité la mise en œuvre par ces acteurs de nouveaux projets d'innovations entre 2006 et 2015 (Fig. 1). L'équipementier a bénéficié en particulier de la nature des savoirs partagés et de la force des relations interpersonnelles. La relation de confiance établie entre les chercheurs et le responsable de l'atelier chez l'équipementier a été centrale dans la réussite du processus. Aujourd'hui, ce technicien est considéré comme un référent, au sein de l'entreprise comme à l'extérieur. Cette confiance constitue selon Medah (2011) la base du travail collectif de conception.

Les situations formelles d'apprentissage sont généralement documentées et en conséquence facilement repérables. Il en va tout autrement des situations non formelles que les chercheurs du domaine technologique n'ont pas l'habitude de renseigner 
(Cerf et Meynard, 2006). Identifier ces situations non formelles d'apprentissage n'est pas aisé, car il s'agit de faire appel à la mémoire des protagonistes sur des faits datant parfois de près de vingt ans. Il a fallu également pister les traces de ces situations dans les différents documents de projet (rapports et comptes rendus de mission).

La construction du récit de l'innovation s'instruit d'abord par l'analyse de documents provenant de la recherche et par l'interview de chercheurs protagonistes du processus. Initialement, la reconstitution de l'histoire de l'innovation s'avère donc marquée par la représentation des chercheurs. Ensuite, les points de vue des acteurs non chercheurs, ici transformatrices et équipementiers, contribuent au façonnage de ce récit. Des interprétations parfois divergentes et des controverses émergent alors, qui contribuent à la production d'un récit contrasté, une des richesses de la méthode ImpresS.

\section{Conclusions}

La construction du récit d'innovation entreprise sur le cas du décortiqueur de fonio révèle une implication de la recherche dans le processus d'innovation qui s'étend sur un temps long de plus de dix ans. La durée de ce processus dépassant considérablement le temps d'un projet de recherche traduit une constance dans les relations entre acteurs majeurs et dans l'implication des chercheurs. Des actions entreprises en amont puis en aval du projet ont ainsi contribué à la mise au point de la solution technique, à la diffusion de l'équipement, favorisant ainsi l'émergence de nouvelles PME de transformation et d'activités de prestation de services.

Cette étude témoigne du rôle déterminant des futures utilisatrices de l'équipement, les transformatrices de fonio. Bien en amont de la phase projet, elles ont interpellé chercheurs et décideurs sur les contraintes de la transformation $\mathrm{du}$ fonio et plus spécifiquement du décorticage. Ces transformatrices incarnent la figure emblématique de l'entrepreneuriat du secteur agroalimentaire en Afrique de l'Ouest; leur implication dans une recherche collaborative a été déterminante. L'utilisation du décortiqueur par ces actrices pivots fut un gage de fiabilité et une condition de la confiance des autres transformatrices; cela contribua également à la crédibilité et à la notoriété de l'équipementier.

La mobilisation de la démarche ImpresS a permis, grâce aux outils tels que la reconstruction du récit de l'innovation et l'analyse des interactions des différentes parties prenantes, de mieux comprendre les raisons du succès de l'innovation «décortiqueur de fonio GMBF». L'étude témoigne de l'importance des apprentissages croisés formels ou informels. Elle montre l'intérêt de la collaboration multi-acteurs dans les processus de conception d'équipements agroalimentaires. C'est là toute la problématique de la conception collaborative au centre de laquelle la connaissance des acteurs de l'innovation joue un rôle majeur.

\section{Références}

Abécassis J, Alause J, Faure J, Feillet P, Gibert F, Lallemant J, et al. 1980. Évaluation comparative des procédés et des équipements industriels de décorticage des céréales, légumineuses et oléagineuses dans le monde. Montpellier: GERDAT-IRAT, 223 p.
Adjei-Nsiah S, Klerkx L. 2016. Innovation platforms and institutional change: the case of small-scale palm oil processing in Ghana. Cahiers Agricultures 25(6): 65005. DOI: 10.1051/cagri/2016046.

Barret D, Blundo-Canto G, Dabat MH, Devaux-Spatarakis A, Faure G, Hainzelin E, et al. 2017. Guide méthodologique ImpresS. Évaluation ex-post des impacts de la recherche agronomique dans les pays du Sud. Montpellier : CIRAD. http://agritrop.cirad.fr/586223.

Bergeret A, Cruz JF, Troude F. 1989. Étude d'une ligne semiindustrielle d'usinage du riz. In: Aupelf-Uref, ed. Céréales en régions chaudes. Paris: John Libbey Eurotext, pp. 231-240.

Blanco E. 1998. L'émergence du produit dans la conception distribuée: vers de nouveaux modes de rationalisation dans la conception de systèmes mécaniques. Thèse de Doctorat en Génie mécanique. Institut National Polytechnique de Grenoble.

Bricas N. 2012. Forces et faiblesses des filières agro-alimentaires en Afrique. Marchés Tropicaux et Méditerranéens 3275-3276: 27-28.

Bricas N, Raoult-Wack AL. 1997. Pour un développement agroalimentaire maîtrisable dans les zones tropicales. Cahiers Agricultures 6(6): 577-589.

Cerf M, Meynard J. 2006. Les outils de pilotage des cultures: diversité de leurs usages et enseignements pour leur conception. Natures Sciences Sociétés 14(1): 19-29.

Cruz JF. 2004. Fonio, a small grain with potential. Leisa. Magazine on low external input and sustainable agriculture 20(1): 16-17. http:// edepot.wur.nl/90002.

Cruz JF, Béavogui F, Dramé D. 2011. Le fonio, une céréale africaine. Collection Agricultures tropicales en Poche. ed. Versailles (France): Quae; Gembloux (Belgique): Presses agronomiques de Gembloux, Wageningen (Pays-Bas): CTA, 175 p.

Cruz JF, Havard M. 1994. Innovations dans le domaine du machinisme agricole tropical. L'exemple du décorticage des céréales en Afrique sub-saharienne. Marchés Tropicaux et Méditerranéens 2539: 1429-1432.

Ferré T, Medah I, Cruz JF, Chtioui M, Dabat M-H., Devaux A. 2016. La mécanisation du décorticage du fonio au Mali et au Burkina Faso. Étude de cas. Montpellier: Cirad. http://agritrop.cirad.fr/ $586750 \%$.

Fofiri Nzossié EJ, Temple L, Ndjouenkeu R. 2015. La contribution de la recherche universitaire à la formation d'un système sectoriel d'innovation agro-alimentaire au Cameroun. Innovations 47(2): $55-77$.

Giroux F, Marouzé C. 2006. Local design capacity building applied to small-scale food processing equipment: a strategic way for adding value to producers. In: CIGR World Congress on Agricultural engineering for a better world, Bonn (Germany), 3-7, September 2006. Book of abstracts. EURAGENG, VDI, FAO. Düsseldorf: Vdi-verlag, pp. 893-894.

Marouzé C. 1999. Proposition d'une méthode pour piloter la trajectoire technologique des équipements dans les pays du Sud. Application au secteur agricole et agroalimentaire. Thèse de doctorat de Génie Industriel. Aix-en-Provence/Paris : ENSAM.

Marouzé C, Diagne A, Giroux F. 2004. Contribution to the building of an equipment design method in developing countries using an analysis of traditional practices. In: Tichkiewitch S, Brissaud D, eds. Methods and tools for co-operative and integrated design. Dordrecht: Kluwer Academic Publishers, pp. 57-70.

Medah I. 2011. Quand l'échange de savoirs se heurte à la reconnaissance. Le cas des concepteurs d'agroéquipements au Burkina Faso. Revue d'anthropologie des connaissances 5(3): 599-622. DOI: 10.3917/rac.014.0599.

Moity-Maïzi P. 2011. Interroger la localisation et la circulation des savoirs en Afrique. Revue d'anthropologie des connaissances 5(3): 473-491. 
Nguena CL. 2013. Solutions pratiques au probleme de financement des PME au Cameroun. ICBE Policy Brief 32: 1-5.

Sardas JC. 1997. Ingénierie intégrée et mutation des métiers de la conception. Annales des Mines - Réalités industrielles 46: 41-48.
Temple L, Biénabe E, Barret D, Saint-Martin G. 2016. Methods for assessing the impact of research on innovation and development in the agriculture and food sectors. African Journal of Science, Technology, Innovation and Development 8(5-6): 399-410.

Citation de l'article : Ferré T, Medah I, Cruz J-F, Dabat M-H, Le Gal P-Y, Chtioui M, Devaux-Spatarakis A. 2018. Innover dans le secteur de la transformation agroalimentaire en Afrique de l'Ouest. Cah. Agric. 27: 15011. 